\title{
SOME NOTES ON EXCEPTIONAL VALUES OF MEROMORPHIC FUNCTIONS
}

\author{
KIKUJI MATSUMOTO
}

1. Let $E$ be a totally-disconnected compact set in the $z$-plane and let $\Omega$ be its complement with respect to the extended $z$-plane. Then $\Omega$ is a domain and we can consider a single-valued meromorphic function $w=f(z)$ on $\Omega$ which has a transcendental singularity at each point of $E$. Suppose that $E$ is a nullset of the class $W$ in the sense of Kametani [4] ( $==$ the class $N_{\mathfrak{B}}$ in the sense of Ahlfors and Beurling [1]). Then the cluster set of $f(z)$ at each transcendental singularity is the whole $w$-plane, and hence $f(z)$ has an essential singularity at each point of $E$. We shall say that a value $w$ is exceptional for $f(z)$ at an essential singularity $\zeta \in E$ if there exists a neighborhood of $\zeta$ where the function $f(z)$ does not take this value $w$.

In our previous paper [6], we showed that, even if $E$ is of capacity ${ }^{11}$ zero, the set of all exceptional values of $f(z)$ at a point $\zeta$ of $E$ may be non-countable. From this fact, there arises the following problem: Is there a perfect set $E$ such that each $f(z)$ has at most a countable number of exceptional values at each essential singularity $\zeta \in E$ ?

Recently, Carleson [3] and the author [7] have given positive answers to this problem, that is, they have given sufficient conditions for sets $E$ to satisty that every $f(z)$ has at most a finite number of exceptional values. Particularly, Carleson's paper is very interesting. Carleson has shown that there exist sets $E$ of positive capacity for which every $f(z)$ has at most three exceptional values. His arguments are based on the fact that a set of linear measure zero is a null-set of the class $W$.

In this note, we shall give a sufficient condition, much better than Carleson's and the author's, using Carleson's arguments essentially.

2. Let $\left\{\Omega_{n}\right\}_{n=0,1,2, \ldots}$ be an exhaustion of $\Omega$ with the following conditions :

Received March 26, 1962.

1) In this note, capacity is always logarithmic. 
1) $\Omega_{n+1} \supset \bar{\Omega}_{n}$ for every $n$,

2 ) for each $n$, the boundary $\partial \Omega_{n}$ of $\Omega_{n}$ consists of a finite number of closed analytic curves,

3) each component of the open set $\mathscr{C} \bar{\Omega}_{n}{ }^{2)}$ contains points of $E$,

4) the open set $\Omega_{n}-\bar{\Omega}_{n-1}(n \geqq 1)$ consists of a finite number of doublyconnected domains $R_{n, k}(k=1,2, \ldots, N(n))$.

We shall use in the sequel the graph associated with $\left\{\Omega_{n}\right\}$ in the sense of Noshiro [9]. Let $u(z)+i v(z)$ be the mapping function of $\Omega-\Omega_{0}$ onto it and let $R$ be its length. ${ }^{3}$

Let $\gamma_{r}$ be the niveau curve $u(z)=r(0<r<R)$ on $\Omega$. The niveau curve $\gamma_{r}$ consists of a finite number of simple closed curves $r_{r, k}(k=1,2, \ldots, n(r))$. We shall call each component of the open set $\Omega_{n}-\bar{\Omega}_{m}(n>m \geqq 0)$ an $R$-chain, consider for every $r_{r, k}(0<r<R, 1 \leqq k \leqq n(r))$ the longest doubly-connected $R$-chain $R\left(\gamma_{r, k}\right)$ such that $\gamma_{r, k}$ is contained in $R\left(\gamma_{r, k}\right)$ or is the one of the two boundary components of $R\left(\gamma_{r, k}\right)$, and denote by $\mu\left(\gamma_{r, k}\right)$ the harmonic modulus of this $R$-chain. We set

$$
\mu(\boldsymbol{r})=\min _{1 \leqq k \leqq n(r)} \mu\left(\gamma_{r, k}\right) .
$$

Generally $R_{n, k}$ may branch off into a finite number of $R_{n+1, m}$. If every $R_{n, k}(n=1,2, \ldots ; k=1,2, \ldots, N(\boldsymbol{n}))$ branches off into at most $\rho$ domains $R_{n+1, m}$, we say that the exhaustion $\left\{\Omega_{n}\right\}$ branches off at most $\rho$-times everywhere.

Now we state our theorem.

THEOREM 1. Let $E$ be a totally-disconnected compact set in the z-plane and let $\Omega$ be its complementary domain. If there exists an exhaustion $\left\{\Omega_{n}\right\}$ of $\Omega$ which satisfies the conditions 1),2),3) and 4) stated above, branches off at most $\rho$-times everywhere and has the graph satisfying the condition that

$$
\lim _{r \rightarrow R} \mu(\boldsymbol{r})=+\infty,
$$

then every function which is single-valued and meromorphic in $\Omega$ and has an

2) We denote the complement of a set $A$ with respect to the extended complex plane by $\mathscr{C} A$.

3) Cf. K. Matsumoto [7], §2. 
essential singularity at each point of $E^{4)}$ has at most $\rho+1$ exceptional values at each singularity.

This is an amelioration of the theorem given in [7]. In fact, we proved the same assertion under the additional conditions that the length $R$ is infinite and further

$$
\varlimsup_{r \rightarrow \infty} \frac{n(r)}{r}<+\infty .
$$

3. We shall prove a little stronger theorem than Theorem 1 . Let $f(z)$ be a single-valued meromorphic function in $\Omega$ possessing at least one essential singularity in $E$, not necessarily at each point of $E$. We shall say that $f(z)$ omits a value $w$ in $\Omega$ at an essential singularity $\zeta \in E$ if there is a neighborhood $U(\zeta)$ of $\zeta$ such that $f(z)$ does not take this value $w$ in $\Omega \cap U(\zeta)$. Such a value may be taken by $f(z)$ only at points of $E$ near $\zeta$.

We shall prove the following

THEOREM 2. Under the same conditions as Theorem 1, every function, which is single-valued and meromorphic in $\Omega$ and has at least one essential singularity in $E$, omits at most $\rho+1$ values in $\Omega$ at each singularity.

In the case where $\rho=1, E$ consists of just one point and hence our assertion is true from Picard's theorem. We shall give a proof only in the case where $\rho=2$. In the same way, we can prove in general cases.

4. Before proving the theorem, we give two lemmas. We shall consider the Riemann sphere $\Sigma$ with radius $1 / 2$ touching the $w$-plane at the origin. For $w$ and $w^{\prime}$ in the $w$-plane we denote by $\left[w, w^{\prime}\right]$ the chordal distance between them, that is,

$$
\left[w, w^{\prime}\right]=\frac{\left|w-w^{\prime}\right|}{\sqrt{\left(1+|w|^{2}\right)\left(1+\left|w^{\prime}\right|^{2}\right)}} .
$$

Further we denote by $C(w ; \delta)(\delta>0)$ the spherical open disc with center at $w$ and with chordal radius $\delta$.

Let $w=f(z)$ be a single-valued meromorphic function in an annulus

4) We shall see in the proof of the theorem that $E$ is a null set of the class $W$ and hence every transcendental singularity of single-valued meromorphic functions in $Q$ is always an essential singularity. 
$1<|z|<e^{\tau}(\sigma>0)$ omitting four values $w_{1}, w_{2}, w_{3}$ and $w_{4}$, and let $\delta>0$ be so small that spherical discs $C\left(w_{i} ; \delta\right)(i=1,2,3,4)$ are mutually disjoint. Now we prove the following lemma which is a consequence of Bohr-Landau's theorem [2]:

If $g(z)$ is regular in $|z|<1$ and $g(z) \neq 0,1$ there, then

$$
\max _{|z|=r}|g(z)| \leqq \exp \left(\frac{K \log (|g(0)|+2)}{1-r}\right) \quad \text { for all } r<1,
$$

where $K$ is a positive constant (a precise form of Schottky's theorem).

Lemma 1. There is a positive constant $\delta^{\prime}$ such that, if $f(z)$ takes a value outside $C\left(w_{i} ; \delta\right)$ for some $i(1 \leqq i \leqq 4)$ at a point on $|z|=e^{\sigma / 2}$, then the image of $|z|=e^{o / 2}$ by $f(z)$ lies completely outside the concentric spherical disc $C\left(w_{i} ; \delta^{\prime}\right)$. Here $\delta^{\prime}$ depends only on $\sigma, w_{i}(1 \leqq i \leqq 4)$ and $\delta$, and does not depend on $f(z)$.

Proof. From Bohr-Landau's theorem, we can see easily that if $w=g(z)$ is a regular function in $1<|z|<e^{\sigma}$ such that

$$
g(z) \neq 0,1 \text { and } \min _{|z|=e^{\sigma / 2}}|g(z)|<M \text { for a positive } M,
$$

then there is a positive constant $M^{\prime}$ depending only on $M$ and $\sigma$ and satisfying

$$
\max _{|z|=e^{\sigma / 2}}|g(z)| \leqq M^{\prime}
$$

We denote by $\zeta=T_{j, m}^{i}(w) \quad(1 \leqq i, j, m \leqq 4, i \neq j, m$ and $j \neq m)$ the linear transformation which transforms $w_{i}, w_{j}$ and $w_{m}$ to the point at infinity, the origin and the point $\zeta=1$ respectively. Since the number of such $T_{j, m}^{i}$ is finite, we can find a positive $M$ so large that, for each $T_{j, m}^{i}$, its image of the outside of $C\left(w_{i} ; \delta\right)$ is contained completely in $|\zeta|<M$. For this $M, \zeta=T_{j, m}^{i}(f(z))$ has the same properties as $g(z)$ stated above, and hence it holds that

$$
\left|T_{j, m}^{i}(f(z))\right| \leqq M^{\prime} \quad \text { on } \quad|z|=e^{o / 2},
$$

where $M^{\prime}>0$ depends only on $M$ and $\sigma$. The image of the outside $V$ of $|\zeta| \leqq M^{\prime}$ by $\left(T_{j, m}^{i}\right)^{-1}$ is an open disc containing $w_{i}$. If we denote by $d_{j, m}^{i}$ the chordal distance between $w_{i}$ and the boundary of $\left(T_{j, m}^{i}\right)^{-1}(V)$ and set

$$
\delta^{\prime}=\min _{\substack{1, i, j, m \leqq 4 \\ i \neq j, m \\ j \neq m}} d_{j, m}^{i},
$$


then $\delta^{\prime}>0$ and obviously satisfies all conditions of the lemma.

Next lemma is a revised form of Carleson's [3].

Lemma 2. Let $w=f(z)$ be a single-valued meromorphic function on an annulus $1 \leqq|z| \leqq e^{\mu}(\mu>0)$. If $f(z)$ takes there no value in a spherical disc $C\left(w_{0} ; \delta\right)$, then there exists a positive constant $A$ depending only on $\delta$ such that the diameter of the image of $|z|=e^{\mu / 2}$ by $f(z)$ with respect to the chordal distance is dominated by $A e^{-\mu / 2}$ for sufficiently large $\mu$.

In particular, if $\delta$ is sufficiently close to 1 , that is, the complementary sherical disc $C\left(-1 / \bar{w}_{0} ; d\right)$ of $C\left(w_{0} ; \delta\right)$ has a radius $d$ sufficiently small, we have

$$
A<B d,
$$

where $B$ is a positive constant.

Proof. We may assume without loss of generality that the center $w_{0}$ of $C\left(w_{0} ; \delta\right)$ is the point at infinity, for otherwise we can transform $w_{0}$ to the point at infinity by the linear transformation $\left(1+\bar{w}_{0} w\right) /\left(w-w_{0}\right)$, under which the chordal distance remains invariant. Let $|w|>M$ be the domain in the $w$-plane corresponding to $C\left(w_{0} ; \delta\right)$. Then

$$
|f(z)| \leqq M \quad \text { on } \quad 1 \leqq|z| \leqq e^{\mu} .
$$

By Cauchy's integral theorem, we have

$$
f^{\prime}(z)=\frac{1}{2 \pi i}\left\{\int_{|\zeta|=e^{\mu}} \frac{f(\zeta)}{(\zeta-z)^{2}} d \zeta-\int_{|\zeta|=1} \frac{f(\zeta)}{(\zeta-z)^{2}} d \zeta\right\}
$$

for every $z$ on $|z|=e^{\mu / 2}$ and hence, if $\mu \geqq 2$,

$$
\left|f^{\prime}(z)\right| \leqq \frac{M}{2 \pi}\left\{\frac{2 \pi e^{\mu}}{\left(e^{u}-e^{\mu / 2}\right)^{2}}+\frac{2 \pi}{\left(e^{u / 2}-1\right)^{2}}\right\} \leqq \frac{2 e^{2}}{(e-1)^{2}} M e^{-\mu} .
$$

Therefore we have

$$
\int_{|z|=e^{u / 2}}\left|f^{\prime}(z)\right||d z| \leqq \frac{2 e^{2}}{(e-1)^{2}} M e^{-\mu} \cdot 2 \pi e^{\mu / 2}=\frac{4 \pi e^{2}}{(e-1)^{2}} M \cdot e^{-\mu / 2} .
$$

The left side shows the length of the image curve $f\left(|z|=e^{\mu / 2}\right)$, and hence the diameter of the image of $|z|=e^{\mu / 2}$ by $f(z)$ with respect to the metric $|d w|$, consequently with respect to the chordal distance, is dominated by $\left(2 \pi e^{2} /\right.$ $\left.(e-1)^{2}\right) M e^{-\mu / 2}$. We can take $\left(2 \pi \varepsilon^{2} /(e-1)^{2}\right) M$ as $A$, for $M$ depends only on $\delta$. 
If $d<1 / 2$, then $M<2 d$. Hence

$$
B=4 \pi e^{2} /(e-1)^{2}
$$

is one of the wanted. Thus our lemma is established.

5. Proof of the theorem. Contrary to our assertion, let us suppose that there exists a function $f(z)$ which is single-valued and meromorphic in $\Omega$, has at least one essential singularity in $E$ and omits more than three values in $\Omega$ at an essential singularity $\zeta_{0} \in E$. Then there is a neighborhood $U\left(\zeta_{0}\right)$ of $\zeta_{0}$ such that $f(z)$ omits four values $w_{1}, w_{2}, w_{3}$ and $w_{i}$ in $U\left(\zeta_{0}\right) \cap \Omega$. We take a positive $\delta$ so small that spherical discs $C\left(w_{i} ; \delta\right)(1 \leqq i \leqq 4)$ are mutually disjoint. For this $\delta$ and a $\sigma>0$, Lemma 1 determines $\delta^{\prime}>0$.

Next we consider this $\delta^{\prime}$ as $\delta$ in Lemma 2 and take $\mu_{0}$ so large that

$$
A e^{-\mu_{0} / 2}<\min \left\{1 / 24, \delta^{\prime} / 3\right\} \text { and } B e^{-\mu_{0} / 2}<1_{1}^{\prime} 12 \text {, }
$$

where $A$ and $B$ are constants in Lemma 2. From the assumption

$$
\lim _{r \rightarrow R} \mu(r)=+\infty
$$

there is an $r_{0}>0$ such that

$$
\mu(r)>\mu_{0}+2 \sigma \quad \text { for all } r: r_{0}<r<R .
$$

The niveau curve $\gamma_{r}: u(z)=r\left(r_{0}<r<R\right)$ consists of a finite number of simple closed curves $\gamma_{r, k}(k=1,2, \ldots, n(r))$, and one of them, say $r r, 1$, encloses $\zeta_{0}$. For $r$ sufficiently near $R$, the longest doubly-connected $R$-chain $R\left(\gamma_{r, 1}\right)=S_{1,1}$ for $\gamma_{r, 1}$, which we defined in $\S 2$, is contained in $U\left(\zeta_{0}\right)$. The harmonic modulus of $S_{1,1}$ is of course greater than $\mu_{0}+2 \sigma$ but is not infinite, for, if so, $\zeta_{0}$ must be isolated and $f(z)$ cannot omit four values at $\zeta_{0}$. Therefore $S_{1,1}$ must branch off. Now suppose that $S_{1,1}$ is a component of the open set $\Omega_{n}-\bar{\Omega}_{n^{\prime}}\left(n>n^{\prime}\right)$, and branches off into two domains $R_{n+1, k}$ and $R_{n+1, k^{\prime}}$, and consider the longest doublyconnected $R$-chains $S_{2,1}$ and $S_{2,2}$ containing $R_{n+1, k}$ and $R_{n+1}$, respectively. Then they have harmonic moduli greater than $\mu_{0}+2 \sigma$, one of them, say $S_{2,1}$, separates $\zeta_{0}$ from $S_{1,1}$ and its harmonic modulus is finite by the same reason as above. Hence $S_{2,1}$ is a component of the open set $\Omega_{m}-\Omega_{n}$ for some $m$ and branches off into two domains $R_{m+1, k}$ and $R_{m+1, k^{\prime}}$. We shall denote by $S_{3,1}$ and $S_{3,2}$ the longest doubly-connected $R$-chains containing them. On the other hand, the harmonic modulus of $S_{2,2}$ may be infinite. If it is infinite, one of the boundary components 
of $S_{2,2}$ is a point $\eta \in E$ and $f(z)$ is meromorphic at $\eta$. If it is finite, we obtain two $R$-chains $S_{3,3}$ and $S_{3,4}$ in the same manner as above. Thus we have at most $2^{2} R$-chains $S_{3, q}$ such that their harmonic moduli are greater than $\mu_{0}+2 \sigma$, one of them encloses $\zeta_{0}$ and each of them branches off into two domains, if its harmonic modulus is finite, or has a point $\eta \in E$ as one of its boundary components at which $f(z)$ is meromorphic, if its harmonic modulus is infinite. Continuing inductively, we obtain a set of $R$-chains $S_{p, q}(\phi=1,2,3, \ldots$; $q=1,2, \ldots, Q(p) \leqq 2^{p-1}$ ) with the following properties:

1) $\bigcup_{p=1}^{\infty} \bigcup_{q=1}^{Q(p)} \bar{S}_{p, q} \supset \Delta$, where $\Delta$ denotes the intersection of the inside of the simple closed curve $\gamma_{r, 1}$ and $\Omega$,

2) their harmonic moduli are greater than $\mu_{0}+2 \sigma$,

3) each $S_{p, q}$ branches off into two $S_{p+1, q}$ if its harmonic modulus is finite, or

4.) it has a point $\eta \in E$ as one of its boundary components and $f(z)$ is meromorphic at $\eta$ if its harmonic modulus is infinite. In this case we shall denote the point $\eta$ and the value $f(\eta)$ by $\eta_{p, q}$ and $w_{p, q}$ respectively.

Each $S_{p, q}$ is conformally equivalent to the annulus $1<|\zeta|<e^{\mu}$, where $\mu$ is the harmonic modulus of $S_{p, q}$. In the case where $\mu<+\infty$, we denote by $S_{p, q}^{1}, S_{p, q}^{2}$ and $S_{p, q}^{3}$ subdomains of $S_{p, q}$ corresponding to the annuli $1<|\zeta|<e^{s}$, $e^{\sigma}<|\zeta|<e^{\mu-\sigma}$ and $e^{\mu-\sigma}<|\zeta|<e^{\mu}$ respectively and by $\Gamma_{p, q}^{1}, \Gamma_{p, q}^{2}$ and $I_{p, q}^{+3}$ closed curves corresponding to $|\zeta|=e^{\sigma / 2},|\zeta|=e^{\mu / 2}$ and $|\zeta|=e^{\mu-\sigma / 2}$ respectively. We observe that for each $I_{p, q}^{2}$ the diameter of its image by $f(z)$ with respect to the chordal distance is dominated by $K=\min \left\{1 / 24, \delta^{\prime} / 3\right\}$. In fact, for $z^{\prime} \in I_{p, q}^{-1}$ and $z^{\prime \prime} \in \Gamma_{p, q}^{3}$, the images $f\left(z^{\prime}\right)$ and $f\left(z^{\prime \prime}\right)$ lie in the outside of at least one $C\left(w_{i} ; \delta\right)$, say $C\left(w_{1} ; \delta\right)$, and hence, applying Lemma 1 in $S_{p, q}^{1}$ and $S_{p, q}^{3}$, we see that the images of $\Gamma_{p, q}^{1}$ and $\Gamma_{p, q}^{3}$, consequently the image of the ring domain bounded by them by the maximum principle, lie completely outside $C\left(w_{1} ; \delta^{\prime}\right)$. Thus our assertion follows from Lemma 2 , because the harmonic modulus of $S_{p, q}^{2}$ is greater than $\mu_{0}$.

Every $S_{p+1, q^{\prime}}(p \geqq 1)$ has in common with another $S_{p+1, q^{\prime \prime}}$ an $S_{p, q}$ branching off into them, and we shall denote by $\Delta_{p, q}$ the triply-connected domain bounded by $I_{p, q}^{2}, \Gamma_{p+1, q^{\prime}}^{2}$ and $\Gamma_{p+1, q^{\prime \prime}}^{2}$, where we consider $\Gamma_{p+1, q^{\prime}}^{2}=\eta_{p+1, q^{\prime}}$ or $I_{p+1, q^{\prime}}^{2}=\eta_{p+1}, q^{\prime \prime}$ if $S_{p+1, q^{\prime}}$ or $S_{p+1, q^{\prime \prime}}$ has infinite harmonic modulus. For $w \in f\left(I_{p, q}^{2}\right), w^{\prime} \in f\left(\Gamma_{p+1, q^{\prime}}^{2}\right)$ and $w^{\prime \prime} \in f\left(\Gamma_{p+1, q}^{2}\right)$ we consider spherical discs $C(w ; K), C\left(w^{\prime} ; K\right)$ and 
$C\left(w^{\prime \prime} ; K\right)$, which of course contain $f\left(\Gamma_{p, q}^{2}\right), f\left(\Gamma_{p+1, q^{\prime}}^{2}\right)$ and $f\left(\Gamma_{p+1, q^{\prime \prime}}^{2}\right)$ respectively. Since $K<\delta^{\prime} / 3$, they cannot contain at least one of $w_{i}(1 \leqq i \leqq 4)$, say $w_{1}$, and hence each one of them cannot be disjoint from the union of the others, for, if so for some one, there is $z_{0} \in \Delta_{p, q}$ such that $f\left(z_{0}\right)$ is contained and can be joined to $w_{1}$ with a curve $A$ in the outside of the union of these three discs, and we are led to a contradiction that the element of the inverse function $f^{-1}$ corresponding to $z_{0}$ can be continued analytically along $A$ up to a point arbitrarily near $w_{1}$ so that $f(z)$ takes the value $w_{1}$ in $\Delta_{p, q}$. Therefore we can conclude that

$\left(1^{\circ}\right)$ for every $\Delta_{p, q}$, there is a spherical disc with the chordal radius $3 \mathrm{~K}$ containing its image $f\left(\Delta_{p, q}\right)$.

Next we shall consider $\Gamma_{p, q}^{2}$ for $p \geqq 2$. Then $\Delta_{p, q}$ and some $\Delta_{p-1, q^{\prime}}$ have $\Gamma_{p, q}^{2}$ as the common boundary and $\Delta_{p, q} \cup \Gamma_{p, q}^{2} \cup \Delta_{p-1, q^{\prime}} \supset S_{p, q}$. From ( $\left.1^{\circ}\right)$ the images of $\Delta_{p, q} \cup I_{p, q}^{\cdot 2} \cup \Delta_{p-1, q^{\prime}}$, consequently of $S_{p, q}^{2} \subset S_{p, q}$, are contained in a spherical disc with the chordal radius $6 K<1 / 2$, so that, applying Lemma 2 in $S_{p, q}^{2}$ for $d=6 K$, we see that the diameter of $f\left(I_{p, q}^{2}\right)<6 K B e^{-\mu_{0} / 2}<K / 2$. For $p \geqq 2$, each boundary component of $\Delta_{p, q}$ thus has the image with diameter less than $K / 2$. By the same reasoning as above we now conclude that

$\left(2^{\circ}\right)$ for $p \geqq 2$, the image of any $\Delta_{p, q}$ is contained in a spherical disc with chordal radius $3 \mathrm{~K} / 2$.

By induction we also see for every $n$ that

$\left(n^{\circ}\right)$ for $p \geqq n$, the image of any $\Delta_{p, q}$ is contained in a spherical disc with chordal radius $3 K / 2^{n-1}$.

Let $\Delta^{\prime}$ be the intersection of the inside of the simple closed curve $\Gamma_{1,1}^{2}$ and $\Omega$ and let $z_{0}$ be a point of $\Gamma_{1,1}^{2}$. Then it follows from the property 1 ) of $\left\{S_{p, q}\right\}$ that

$$
\bigcup_{p=1}^{\infty} \bigcup_{q=1}^{Q(p)} \bar{J}_{p, q} \supset \Delta^{\prime}
$$

and hence, for any $z^{\prime} \in \Delta^{\prime}$, there is $\Delta_{p^{\prime}, q^{\prime}}$ whose closure contains $z^{\prime}$. From $\left(n^{\circ}\right)$ obtained above, we have for a chain of $\Delta_{p, q}$ joining $\Delta_{1,1}$ and $\Delta_{p^{\prime}, q^{\prime}}$

$$
\begin{aligned}
{\left[f\left(z^{\prime}\right), f\left(z_{0}\right)\right] } & \leqq \sum_{p=1}^{p^{\prime}} \text { diam. of } f\left(\Delta_{p, q}\right) \text { w.r.t. the chordal distance } \\
& <2 \sum_{p=1}^{\infty} 3 K / 2^{p-1}=12 K<1 / 2 .
\end{aligned}
$$

By means of a linear transformation we can consider from the above that 
$f(z)$ is bounded in $4^{\prime}$. On the other hand, only applying Pfluger-Mori's criterion ( $[10],[8]$ ) to the ring domains $\left\{S_{p, q}\right\}$, we can see easily that the part $E^{\prime}$ of $E$ contained in the inside of $\Gamma_{1,1}^{2}$ is a null-set of the class $W$. Hence each point of $E^{\prime}$ must be a removable singularity of a bounded function $f(z)$. This contradicts our assumption that $\zeta_{0} \in E^{\prime}$ is an essential singularity of $f(z)$, and hence $f(z)$ cannot omit four values in $\Omega$ at $\zeta_{0}$. Thus our theorem is proved completely.

6. For Cantor sets, we have the following which is an immediate consequence of our theorem.

ThEOREM 3. Let $E$ be a Cantor set on the closed interval $[0,1]$ with the successive ratios $\xi_{n}$ satisfying the condition

$$
\lim _{n \rightarrow \infty} \xi_{n}=0 .
$$

Then every function, which is single-valued and meromorphic in $\Omega=\mathscr{C} E$ and has at least one essential singularity in $E$, omits at most three values in $\Omega$ at each essential singularity.

Carleson proved in his paper [3] the same assertion under a stronger condition

$$
\lim _{n \rightarrow \infty} \frac{\log \xi_{n}^{-1}}{\log n}=+\infty
$$

As he remarked there, there is a set $E$ of positive capacity because $E$ is of capacity zero if and only if

$$
\sum_{n=1}^{\infty} \frac{\log \xi_{n}^{-1}}{2^{n}}=+\infty
$$

7. In our paper [7], we showed, by using its Theorem 1 , that there is a set $E$ such that all $f(z)$ possessing $E$ as the set of essential singularities have at most three exceptional values and some one of them indeed has just three exceptional values at each point of $E$. But the condition

$$
\varlimsup_{r \rightarrow \infty} \frac{n(r)}{r}<+\infty,
$$

which gave at that time some difficulties in constructing $E$, is unnecessary as we saw above and so for each $\rho \geqq 2$ we can give easily in the similar manner 
a set $E$ such that all $f(z)$ have at most $\rho$ exceptianal values and some one of them indeed has just $\rho$ exceptional values.

8. In the last section we shall be concerned with single-valued meromorphic functions which have as the set of essential singularities a set $E$ satisfying the conditions of Theorem 1 for $\rho=2$ and have three exceptional values at a point $\zeta \in E$. We begin with

Lemma 3. Let $f(z)$ be a single-valued meromorphic function on the closure of a triply-connected domain $\Delta$ omitting three values $w_{1}, w_{2}$ and $w_{3}$ there, let $\Gamma_{1}, \Gamma_{2}$ and $\Gamma_{3}$ be the boundary components of $\Delta$ and let $\delta>0$ be so small that the discs $C\left(w_{i} ; \delta\right)(1 \leqq i \leqq 3)$ are mutually disjoint. If $f\left(\Gamma_{i}\right) \subset C\left(w_{i} ; \delta\right)$ for all $i, f(z)$ takes in $\Delta$ each value outside $\bigcup_{i=1}^{3} C\left(w_{i} ; \delta\right)$ once and only once.

Proof. Contrary, suppose that $f(z)$ takes a value $w_{0}$ outside $\bigcup_{i=1}^{3} C\left(w_{i} ; \delta\right)$ at two points $z^{\prime} \in \Delta$ and $z^{\prime \prime} \in \Delta$. We can join $w_{0}$ to $C\left(w_{1} ; \delta\right)$ and $C\left(w_{2} ; \delta\right)$ with curves $\Lambda_{1}$ and $\Lambda_{2}$, respectively, which lie outside $\bigcup_{i=1}^{3} C\left(w_{i} ; \delta\right)$, do not intersect each other except at $w_{0}$ and do not pass through any projection of branch points of the Riemannian image of $\Delta$ by $f(z)$. The elements of the inverse function $f^{-1}$ corresponding to $z^{\prime}$ and $z^{\prime \prime}$ can be continued analytically along these curves to their end points and further from them along radii of $C\left(w_{1} ; \delta\right)$ and $C\left(w_{2} ; \delta\right)$ so that the curves in $\Delta$ corresponding to these continuations join each of $z^{\prime}$ and $z^{\prime \prime}$ to $I_{1}$ and $\Gamma_{2}$ and with parts of $\Gamma_{1}$ and $\Gamma_{2}$ bound a domain not containing $\Gamma_{3}$. Since $\Delta$ has no boundary other than $\Gamma_{1}$, $\Gamma_{2}$ and $\Gamma_{3}$, this domain must be a subdomain of $\Delta$ and $f(z)$ must take the value $w_{3}$ in it; this contradiction proves the lemma.

Let $E$ be a compact set in the $z$-plane satisfying the conditions of Theorem 1 for $\rho=2$ and let $f(z)$ be a single-valued meromorphic function which has $E$ as the set of essential singularities. Of course, $f(z)$ has at most three exceptional values at any point of $E$. From Kuroda's theorem [5] we can easily see that the complementary domain $\Omega$ of $E$ belongs to the class $O_{A B}^{\circ}$ as a Riemann surface and hence the covering surface, which is the Riemannian image of $\Omega$ by $w=f(z)$, has Inversen's property. Consequently, any exceptional value $\alpha$ of $f(z)$ at an essential singularity $\zeta \in E$ is an asymptotic value at $\zeta$ or there is a sequence $\left\{\zeta_{n}\right\}$ of points of $E$ such that $\lim _{n \rightarrow \infty} \zeta_{n}=\zeta$ and $\alpha$ is an asymptotic 
value at every $\zeta_{n}$. Now, suppose that $f(z)$ has indeed three exceptional values $w_{1}, w_{2}$ and $w_{3}$ at $\zeta \in E$. We prove

THEOREM 4. There exists a sequence $\left\{\zeta_{n}\right\}$ of points of $E$ such that $\lim _{n \rightarrow \infty} \zeta_{n}=\zeta$ and for any curve terminating at $\zeta_{n}$ the cluster set of $f(z)$ along it contains always all exceptional values $w_{1}, w_{2}$ and $w_{3}$ ai $\zeta$.

Proof. It is sufficient to prove that in any neighborhood $U(\zeta)$ of $\zeta$ there is a $\zeta^{\prime} \in E$, different from $\zeta$, having the property stated in the theorem. We shall take $\delta>0$ so small that $\operatorname{discs} C\left(w_{i} ; 2 \delta\right)(1 \leqq i \leqq 3)$ are mutually disjoint, use the notations in $\S 5$ considering $2 \delta$ as $\delta$ there and note that in the present case all $S_{p, q}$ branch off into two $S_{p+1 . q}$, because every point of $E$ is an essential singularity of $f(z)$. Further, we can prove by the same reasoning as used in $\S 5$ that if $f\left(\Delta_{p, q}\right)$ lies completely outside some $C\left(w_{i} ; \delta\right)$, then there is a spherical disc $C_{p, q}$ with the chordal radius $3 K$ containing $f\left(\Delta_{p, q}\right)$. In fact, if one of the spherical discs $C(w ; K), C\left(w^{\prime} ; K\right)$ and $C\left(w^{\prime \prime} ; K\right)$ $\left(w \in f\left(\Gamma_{p, q}^{2}\right), w^{\prime} \in f\left(\Gamma_{p+1, q^{\prime}}^{2}\right)\right.$ and $\left.w^{\prime \prime} \in f\left(\Gamma_{p+1, q^{\prime \prime}}^{2}\right)\right)$ is disjoint from the union of the others, there is a point $z_{0} \in A_{p, q}$ with the image $f\left(z_{0}\right)$ outside these three discs, $f\left(z_{0}\right)$ can be joined to the center $w_{i}$ of $C\left(w_{i} ; \delta\right)$ with a curve $A$ outside them and hence $f(z)$ takes the value $w_{i}$ in $\Delta_{p, q}$; this is a contradiction. Thus if, for every $\Delta_{p, q}$ there is one in $C\left(w_{i} ; \delta\right)(1 \leqq i \leqq 3)$ which is disjoint from $f\left(\Delta_{p, q}\right)$, then the assertion $\left(1^{\circ}\right)$ in $\S 5$ holds and we can repeat the arguments there to reach the contradiction that all points of $E$ near $\zeta$ are removable singularities of $f(z)$. Thus we can conclude that there is an infinite number of $\Delta_{p, q}$ such that three discs $C\left(w_{i} ; 2 \delta\right)(1 \leqq i \leqq 3)$ contain the images of its three boundary components one by one.

Let $\delta$, be a sequence of positive numbers decreasing to zero. Then from the above there is a $\Delta_{p, q}$ such that three discs $C\left(u_{i}^{\prime} ; 2 \delta_{1}\right)$ contain the images of its three boundary components one by one. We denote by $\Gamma_{0}$ the boundary component of $\Delta_{p, q}$ with the image $f\left(\Gamma_{0}\right)$ contained in $C\left(w_{1} ; 2 \delta_{1}\right)$ and consider all $\Delta_{p, q}$ contained in the inside of $\Gamma_{0}$. Among these $\Delta_{p, q}$ there is one such that three discs $C\left(w_{i} ; 2 \delta_{2}\right)$ contain the images of its three boundary components one by one and we denote by $\Gamma_{1}$ its boundary component with the image $f\left(\Gamma_{1}\right)$ contained in $C\left(w_{2} ; 2 \delta_{2}\right)$. Next we consider all $\Delta_{p}, q$ contained in the inside of $\Gamma_{1}$ and obtain $\Gamma_{2}$ with the image $f\left(\Gamma_{2}\right)$ contained in $C\left(w_{3} ; 2 \delta_{3}\right)$. We 
proceed inductively and obtain a sequence of closed curves $\left\{\Gamma_{\nu}\right\}$ with the following conditions: 1 ) the inside of $\Gamma_{\nu}$ contains $\left.\Gamma_{\nu+1}, 2\right)$ the image of $I_{3 \kappa+\tau}$ $(\kappa=0,1,2, \ldots ; \tau=0,1,2)$ is contained in $C\left(w_{\tau+1} ; 2 \delta_{3 \kappa+\tau}\right)$. We set

$$
\zeta^{\prime}=\bigcap_{\nu} \overline{\left(\Gamma_{\nu}\right)}
$$

where we denote by $\left(\Gamma_{\nu}\right)$ the inside of $\Gamma_{\nu}$ and by $\overline{\left(\Gamma_{\nu}\right)}$ the closure of $\left(\Gamma_{\nu}\right)$. Obviously $\zeta^{\prime} \in E$ and has the property stated in the theorem. Our proof is now complete.

The fact in the above proof that for any $\delta>0$, there is $\Delta_{p, q}$ such that three discs $C\left(w_{i} ; \delta\right)$ contain the images of its three boundary components one by one implies by Lemma 3 the following theorem under the same conditions as Theorem 4.

THEOREM 5. Let $\Phi$ be the covering surface of the w-plane which is the Riemannian image of $\Omega$ by $w=f(z)$. Then, for arbitrary four discs $C_{i}(1 \leqq i \leqq 4)$ in the w-plane with the closures being mutually disjoint, there is at least one over which $\$$ has an infinite number of univalent discs $\left\{\widehat{C}_{k}\right\}$ such that $f^{-1}\left(\widetilde{C}_{k}\right)$ are compact relative to $\Omega$ and are contained in any neighborhood of $\zeta$ except for a finite number of them.

\section{REFERENCES}

[1] L. V. Ahlfors and A. Beurling: Conformal invariants and function-theoretic nullsets, Acta Math., 83 (1950), pp. 101-129.

[2] H. Bohr and E. Landau: Über das Verhalten von $\zeta(s)$ und $\zeta_{k}(s)$ in der Nähe der Geraden $\sigma=1$, Göttinger Nachr., (1910).

[ 3 ] L. Carleson: A remark on Picard's theorem, Bull. Amer. Math. Soc., 67 (1961), pp. $142-144$.

[ 4 ] S. Kametani: On Hausdorff's measures and generalized capacities with some of their applications to the theory of functions, Jap. Journ. Math., 19 (1944-48), pp. 217-257.

[5] T. Kuroda: On analytic functions on some Riemann surfaces, Nagoya Math. Journ., 10 (1956), pp. 27-50.

[6] K. Matsumoto: Exceptional values of meromorphic functions in a neighborhood of the set of singularities, Journ. Sci. Hiroshima Univ, A 24 (1960), pp. 143-153.

[7] K. Matsumoto: On exceptional values of meromorphic functions with the set of singularities of capacity zero, Nagoya Math. Journ., 18 (1961), pp. 171-191.

[8] A. Mori: A note on unramified abelian covering surfaces of a closed Riemann surface, Journ. Math. Soc. Japan, 6 (1954), pp. 162-176.

[9] K. Noshiro: Open Riemann surface with null boundary, Nagoya Math. Journ., 3 (1951), pp. 73-79. 
[10] A. Pfluger: Sur l'existence de fonctions non constantes, analytiques, conformes et bornées sur une surface de Riemann ouverte, C. R. Paris, 230 (1950), pp. 166-168.

Mathematical Institute,

Nagoya University 